\title{
Structural and Optical Inhomogeneities of Fe doped GaN grown by Hydride Vapor Phase Epitaxy
}

\author{
E. Malguth, ${ }^{1, *}$ A. Hoffmann, ${ }^{1}$ and M. R. Phillips ${ }^{2}$ \\ ${ }^{1}$ Institut für Festkörperphysik, Technische Universität Berlin, \\ Hardenbergstraße 36, 10623 Berlin, Germany \\ ${ }^{2}$ Microstructural Analysis Unit, University of Technology, \\ Sydney, Broadway, NSW 2007 Australia
}

(Dated: September 30, 2008)

\begin{abstract}
We present the results of cathodoluminescence experiments on a set of Fe doped GaN samples with Fe concentrations of $5 \times 10^{17} \mathrm{~cm}^{-3}, 1 \times 10^{18} \mathrm{~cm}^{-3}, 1 \times 10^{19} \mathrm{~cm}^{-3}$ and $2 \times 10^{20} \mathrm{~cm}^{-3}$. These specimens were grown by hydride vapor phase epitaxy with different concentrations of Fe. The introduction of $\mathrm{Fe}$ is found to promote the formation of structurally inhomogeneous regions of increased donor concentration. We detect a tendency of these regions to form hexagonal pits at the surface. The locally increased carrier concentration leads to enhanced emission from the band-edge and the internal ${ }^{4} \mathrm{~T}_{1}(\mathrm{G})-{ }^{6} \mathrm{~A}_{1}(\mathrm{~S})$ transition of $\mathrm{Fe}^{3+}$. In these areas, the luminescence forms a finely structured highly symmetric pattern, which is attributed to defect migration along strain-field lines. Fe doping is found to quench the yellow defect luminescence band and to enhance the blue luminescence band due to lowering of the Fermi level and the formation of point defects, respectively.
\end{abstract}

PACS numbers: 12345 


\section{INTRODUCTION}

In addition to $\mathrm{Mn}$ doped GaN, Fe doped GaN is also attracting increasing interest as a dilute magnetic semiconductor for spin-based applications ${ }^{1}$. The Fe-related electronic states in GaN have recently been established ${ }^{2-5}$. However, the growth of GaN with large concentrations of $\mathrm{Fe}$, which are required for carrier mediated spin-coupling, still poses a challenge. In order to shed light on the effects of Fe doping on the structural and electronic properties of GaN, we carried out cathodoluminescence (CL) experiments on a set of GaN samples with varying Fe concentration from $2 \times 10^{17} \mathrm{~cm}^{-3}$ to $2 \times 10^{20} \mathrm{~cm}^{-3}$. Our results reveal structural and electronic inhomogeneities that lead to a spatial contrast in form of a fine highly symmetric pattern in CL images.

\section{EXPERIMENTAL DETAILS}

A set of Fe doped GaN samples was grown using hydride vapour phase epitaxy (HVPE) on sapphire substrate.

The epilayers are of wurtzite crystal structure with the c-axis aligned to the growth direction. Iron was incorporated during the growth process at concentrations of $5 \times 10^{17} \mathrm{~cm}^{-3}$, $1 \times 10^{18} \mathrm{~cm}^{-3}, 1 \times 10^{19} \mathrm{~cm}^{-3}$ and $2 \times 10^{20} \mathrm{~cm}^{-3}$ as determined by secondary ion mass

spectroscopy (SIMS). The substrate was removed resulting in $\sim 400 \mu \mathrm{m}$ thick free standing samples.

CL experiments were carried out using a LEO Supra VP scanning electron microscope (SEM) equipped with a Gatan MonoCL2 system. A liquid helium cold stage facilitated temperatures down to $5 \mathrm{~K}$. The light was detected by a Peltier cooled Hamamatsu R94302 PMT UV-Vis detector or by a liquid nitrogen cooled Hamamatsu R-5509-72 PMT IR detector.

\section{RESULTS AND DISCUSSION}

\section{A. Structural and Electronic Nonuniformity}

SEM images of the Fe doped GaN samples recorded at $80 \mathrm{~K}$ with an acceleration voltage of $20 \mathrm{kV}$ exhibit mild charging. For all investigated Fe concentrations, isolated regions 
of decreased charging were observed on the (0001) surface (Fig. 1(a)), indicating locally enhanced conductivity. This behavior is particularly evident in the area in and around hexagonal pits, which are found on the surface. In CL experiments (Figs. 1(b) and 2), the band-edge luminescence in these regions was found to be enhanced by up to four orders of magnitude as compared with the regular surface. This effect can not be explained by charging alone. Indeed the CL spectra of "bright" and "dark" regions show other significant differences (Fig. 2) as well (see below).

In the literature, such a spatial contrast of band-edge emission intensity in GaN is attributed to structural inhomogeneities that induce doping inhomogeneities, leading to spatially varying carrier concentration ${ }^{6-11}$. Experimental and theoretical studies ${ }^{6,12,13}$ demonstrate that increased electron concentration in GaN leads to a broad band-edge CL of enhanced intensity with the maximum shifted to higher energies. In the dark area, the band-edge luminescence can be associated with excitonic recombination ${ }^{12,14-17}$, while, in the bright regions, it is dominated by free-carrier-recombination indicating degenerate n-type GaN (Fig. 2). The extension to high energies observed for Fe concentrations of $1 \times 10^{18} \mathrm{~cm}^{-3}$ and $1 \times 10^{19} \mathrm{~cm}^{-3}$ can be explained by the Moss-Burstein effect ${ }^{12,13}$ induced by a locally increased donor concentration resulting from structural inhomogeneities in this region. The increased electron concentration also explains the locally reduced charging effects seen in Fig. 1(a).

According to the literature, those structurally inhomogeneous regions are the top ends of columnar domains of high defect density which originate from the epilayer-substrate interface and extend through to the surface ${ }^{6,8,18-20}$. In HVPE grown GaN, these regions are prone to form pits such as the one depicted in Fig. 1(a) $)^{6,7,18,21,22}$. They consist of $\left\{\begin{array}{llll}1 & 1 & \overline{2} & 2\end{array}\right\}$ facets. The exact mechanisms behind the formation of the pits are still unknown. Motoki et al.associate the pits with local regions of N-polarity with a lower growth rate than the surrounding Ga-polar material ${ }^{23,24}$. For our samples we found the pit density to scale with the Fe concentration. The same observation was made for Ni doping ${ }^{25}$. We attribute the increased tendency of pit formation to strain induced by transition metal doping. Smaller degrees of strain found inside the pits confirm that their formation is associated with the release of strain $^{21}$.

Another result of our CL experiments is that the intrinsic yellow luminescence of GaN around $2.2 \mathrm{eV}$ is quenched by the incorporation of Fe. This can be explained by a lowered 
Fermi level caused by the deep $\mathrm{Fe}^{3+/ 2+}$ acceptor. As a result, the native donor states involved in the YL become ionised. The mechanisms behind the yellow luminescence involving shallow and deep double donors are elucidated in Ref. ${ }^{26}$.

Moreover, the blue band around $2.9 \mathrm{eV}$ shows up for $\mathrm{Fe}$ concentrations above $1 \times 10^{19} \mathrm{~cm}^{-3}$. At such a high impurity concentration, there is a general degradation of crystal quality promoting the formation of donor-acceptor-pairs that are known to be responsible for the $\mathrm{BL}^{27,28}$.

\section{B. CL Pattern}

The bright region displayed in Fig. 1(b) reveals a finely structured, highly symmetric CL pattern under low-voltage excitation. Fig. 3 displays a panochromatic CL image taken of the GaN:Fe sample with $[\mathrm{Fe}]=1 \times 10^{19} \mathrm{~cm}^{-3}$. Similar patterns are found in pits in

the other Fe-doped samples, too. Two other different types of circular features found in the $[\mathrm{Fe}]=1 \times 10^{19} \mathrm{~cm}^{-3}$ sample are presented in Fig. 4. The panchromatic CL images presented in Figs. 3 and 4 were recorded in panchromatic mode, however they mainly show the CL distribution of the dominate band-edge luminescence. Figs. 3(c) and (d) confirm the CL pattern is stable against temperature variations between $25 \mathrm{~K}$ and $300 \mathrm{~K}$. With rising acceleration voltage $\left(\mathrm{E}_{0}\right)$ the image details blur and the contrast fades. This observation demonstrates that the CL pattern is a surface effect as the CL is excited deeper in the sample at higher acceleration voltage.

The origin of the symmetric CL pattern is unclear. Possible contributing mechanisms are symmetric arrangements of structural and doping nonuniformities, strain effects and optical interference.

The CL patterns observed inside the hexagonal pits show a sixfold symmetry correlating with that of the pit. Moreover, axes of mirror symmetry run down the $\left\{\begin{array}{llll}1 & 1 & \overline{2} & 2\end{array}\right\}$ facets of the pits (e.g., Fig. 3(d)). The axis itself appears as a chain of small periodically spaced bright dots where the dark lines terminate as well as dark dots and dashes where there is no correlating structural feature found in the SE image. Figure 5 shows monochromatic images of the axis at two different emission energies, 3.43 and $3.46 \mathrm{eV}$. These data indicate that the maximum of the band-edge luminescence is shifted to smaller energies in spots along the axis. Such a shift indicates that strain may play an important role in the observed CL 
contrast in the pits where the bright spots represent regions of localized tensile strain. One possible scenario is that the donor-like defects that are responsible for the enhanced bandedge luminescence are arranged along symmetric strain-field lines within the pit. Tilting and rotation experiments have ruled out optical interference effects.

\section{The $\mathrm{Fe}^{3+}$ Luminescence}

Figure 6 presents infrared CL spectra of GaN with a Fe concentration of $1 \times 10^{18} \mathrm{~cm}^{-3}$. The complex luminescence structure starting at $1.3 \mathrm{eV}$ represents the radiative intra-center ${ }^{4} \mathrm{~T}_{1}(\mathrm{G})-{ }^{6} \mathrm{~A}_{1}(\mathrm{~S})$ transition of $\mathrm{Fe}^{3+}$ on Ga-site ${ }^{3}$. All investigated Fe doped GaN samples exhibit this emission feature confirming that Fe is successfully incorporated on Ga-site.

Monochromatic CL images with the light detection tuned to the $\mathrm{Fe}^{3+}$ emission at $1.3 \mathrm{eV}$ are displayed in Fig. 7. The $\mathrm{Fe}^{3+}$ luminescence distribution shows the same spatial contrast as the band-edge emission (Figs. 1 and 4) with enhanced intensity in circular areas and hexagonal pits where an increased defect and carrier density is found. This correlation can be explained by the increased donor densities in those regions. The generation of free holes via native defects in GaN acting as donors is known to be an efficient excitation mechanism for $\mathrm{Fe}^{3+3}$.

\section{Conclusion}

The incorporation of Fe into GaN was found to quench the native YL of GaN which could be explained by a lowered Fermi level. The presence of Fe also induces the formation structurally and electronically nonuniform regions of higher donor concentration. This seems to be a general phenomenon of TM doped HVPE-grown GaN. The formation of hexagonal pits consisting of $\left\{\begin{array}{llll}1 & 1 & \overline{2} & 2\end{array}\right\}$ facets is observed in those regions. The enhanced emission in these regions shows a finely structured highly symmetric pattern near the surface under low$\mathrm{kV}$ irradiation. This CL contrast has been attributed to defects localizing along strain-fields within the pits. The radiative ${ }^{4} \mathrm{~T}_{1}(\mathrm{G})-{ }^{6} \mathrm{~A}_{1}(\mathrm{~S})$ transition of $\mathrm{Fe}^{3+}$ on $\mathrm{Ga}$ site was observed at $1.3 \mathrm{eV}$ in all investigated GaN:Fe samples. Also the intensity of this luminescence is enhanced in the region of increased carrier density. 


\section{ACKNOWLEDGMENT}

The authors would like to thank Prof. Dominique Drouin for access to the Zeis Supra and MonoCL2 at the University of Sherbrooke, Canada.

* corresponding author. E-mail address: Malguth@physik.tu-berlin.de; also at Microstructural Analysis Unit, University of Technology, Sydney, Australia

1 A. Bonanni, M. Kiecana, C. Simbrunner, T. Li, M. Sawicki, M. Wegscheider, M. Quast, H. Przybylinska, A. Navarro-Quezada, R. Jakiela, et al., Phys. Rev. B 75, 125210 (2007).

2 E. Malguth, A. Hoffmann, and M. R. Phillips, Phys. Status Solidi B 245, 455 (2008).

${ }^{3}$ E. Malguth, A. Hoffmann, W. Gehlhoff, O. Gelhausen, M. R. Phillips, and X. Xu, Phys. Rev. B 74, 165202 (2006).

4 E. Malguth, A. Hoffmann, and X. Xu, Phys. Rev. B 74, 165201 (2006).

5 E. Malguth, A. Hoffmann, S. Werner, M. H. Kane, and I. T. Ferguson, Mat. Res. Soc. Symp. Proc. 1040, Q06 (2008).

6 T. Paskova, E. M. Goldys, and B. Monemar, J. Cryst. Growth 203, 1 (1999).

7 E. M. Goldys, M. Godlewski, R. Langer, and A. Barski, Appl. Surf. Sci. 153, 143 (2000).

8 M. Godlewski, E. M. Goldys, M. R. Phillips, R. Langer, and A. Barski, Appl. Phys. Lett. 73, 3686 (1998).

9 F. A. Ponce, D. P. Bour, W. Gotz, and P. J. Wright, Appl. Phys. Lett. 68, 57 (1996).

10 S. J. Rosner, E. C. Carr, M. J. Ludowise, G. Girolami, and H. I. Erikson, Appl. Phys. Lett. 70, 420 (1997).

11 M. Godlewski, E. M. Goldys, M. R. Phillips, K. Pakula, and J. M. Baranowski, Appl. Surf. Sci. $177,22(2000)$.

12 B. Arnaudov, T. Paskova, E. M. Goldys, R. Yakimova, S. Evtimova, I. G. Ivanov, A. Henry, and B. Monemar, J. Appl. Phys. 85, 7888 (1999).

13 B. Arnaudov, T. Paskova, E. M. Goldys, S. Evtimova, and B. Monemar, Phys. Rev. B 64, 045213 (2001).

14 R. Dingle, D. D. Sell, S. E. Stokowski, and M. Ilegems, Phys. Rev. B 4, 1211 (1971).

15 O. Lagerstedt and B. Monemar, J. Appl. Phys. 45, 2266 (1974). 
16 J. Menniger, U. Jahn, O. Brandt, H. Yang, and K. Ploog, Phys. Rev. B 53, 1881 LP (1996).

17 J. F. Muth, J. H. Lee, I. K. Shmagin, R. M. Kolbas, H. C. Casey, Jr., B. P. Keller, U. K. Mishra, and S. P. DenBaars, Appl. Phys. Lett. 71, 2572 (1997).

18 J. Kim and K. H. Baik, J. Ceram. Process. Res. 8, 277 (2007).

19 E. M. Goldys, T. Paskova, I. G. Ivanov, B. Arnaudov, and B. Monemar, Appl. Phys. Lett. 73, 3583 (1998).

20 M. Godlewski, E. M. Goldys, G. Pozina, B. Monemar, K. Pakula, J. M. Baranowski, P. Prystawko, and M. Leszczynski, Physica B 308-310, 102 (2001).

21 T. B. Wei, P. Ma, R. F. Duan, J. X. Wang, J. M. Li, Z. Liu, and Y. P. Zeng, Mater. Lett. 61, 3882 (2007).

22 E. M.Goldys, M. Godlewski, T. Paskova, G. Pozina, and B. Monemar, MRS Internet J. N. S. R. 6 (2001).

23 K. Motoki, T. Okahisa, S. Nakahata, N. Matsumoto, H. Kimura, H. Kasai, K. Takemoto, K. Uematsu, M. Ueno, Y. Kumagai, et al., J. Cryst. Growth 237-239, 912 (2002).

${ }^{24}$ K. Motoki, T. Okahisa, R. Hirota, S. Nakahata, K. Uematsu, and N. Matsumoto, J. Cryst. Growth 305, 377 (2007).

25 publication in preparation (????).

26 A. Hoffmann, L. Eckey, P. Maxim, J.-C. Holst, R. Heitz, D. M. Hofmann, D. Kovalev, G. Steude, D. Volm, B. K. Meyer, et al., Solid State Electron. 41, 275 (1997).

27 M. Albrecht, H. P. Strunk, J. L. Weyher, I. Grzegory, S. Porowski, and T. Wosinski, J. Appl. Phys. 92, 2000 (2002).

28 M. A. Reshchikov and H. Morkoc, J. Appl. Phys. 97, 061301 (2005). 


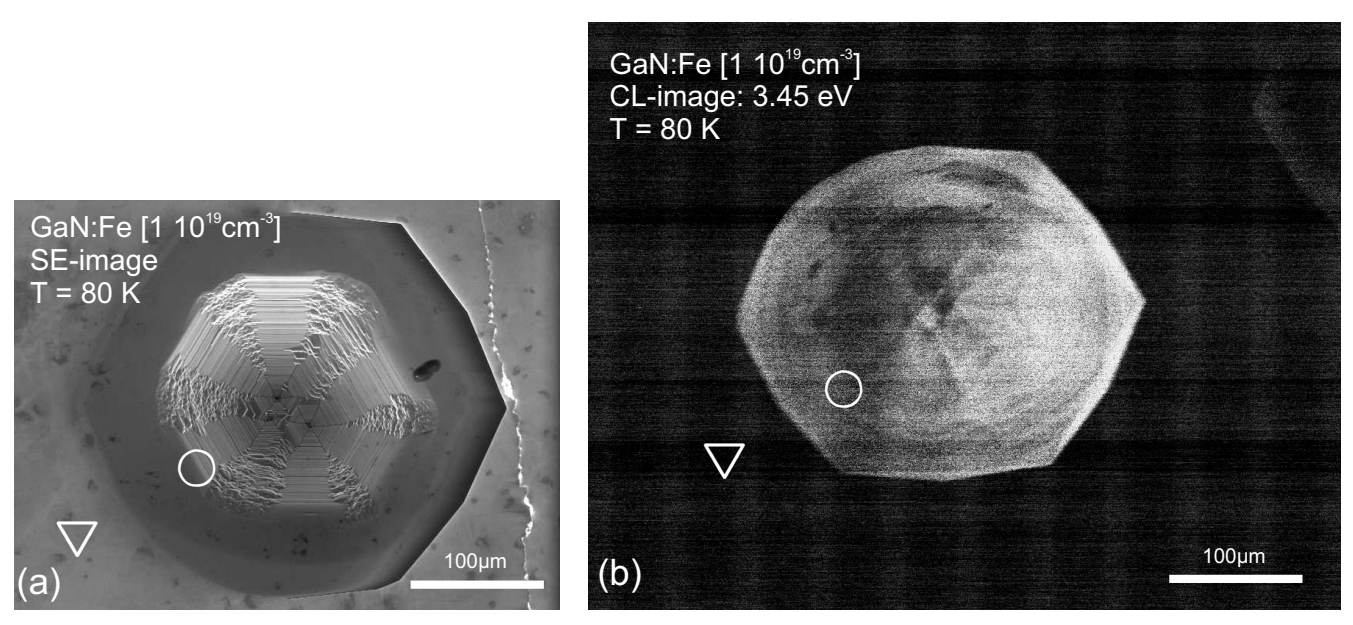

FIG. 1: SE (a) and CL image (b) of Fe doped $\mathrm{GaN}\left([\mathrm{Fe}]=1 \times 10^{19} \mathrm{~cm}^{-3}\right)$ taken at $80 \mathrm{~K}$ with an acceleration voltage of $20 \mathrm{kV}$ and a beam current of $5 \mathrm{nA}$. Both images show the same area of the sample's surface, two hexagonal pits merged into one. A well-defined area of decreased electron emission efficiency around the pit is observed in the SE image (a). The CL image (b) reveals a strongly enhanced band-edge luminescence in this area including the pit. The symbols $\nabla$ and $\bigcirc$ indicate where the spectra in Figs. 2 and 6 were recorded. 


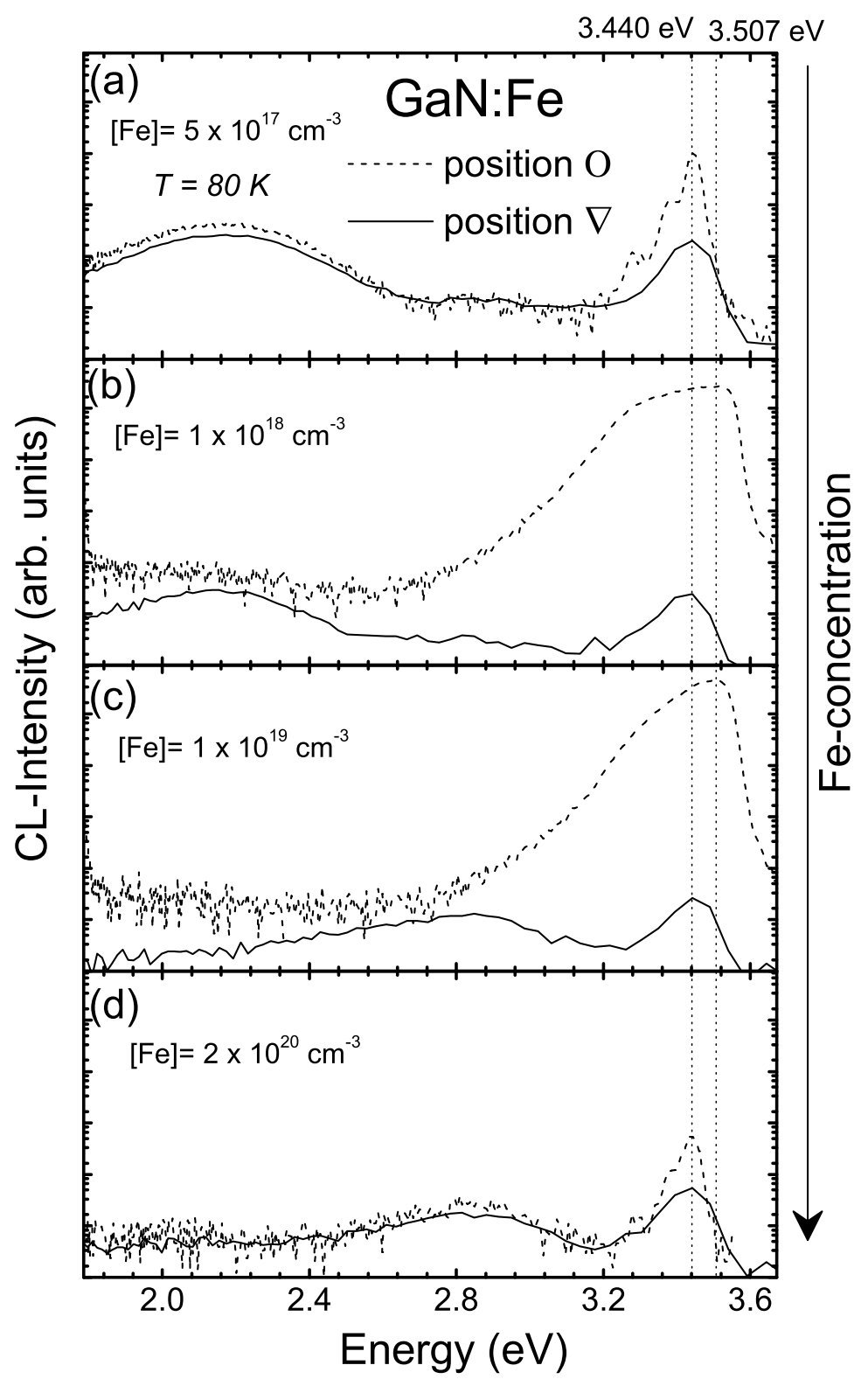

FIG. 2: CL spectra of GaN doped with varying concentrations of Fe at $80 \mathrm{~K}$ on logarithmic scale. Acceleration voltage and current were $20 \mathrm{kV}$ and $5 \mathrm{nA}$, respectively. The symbols $\nabla$ and $\bigcirc$ refer to Fig. 1 and indicate where the spectra were recorded, i.e., areas of strong or weak band-edge luminescence, respectively. The spectra are displayed on the same energy and intensity scales. 

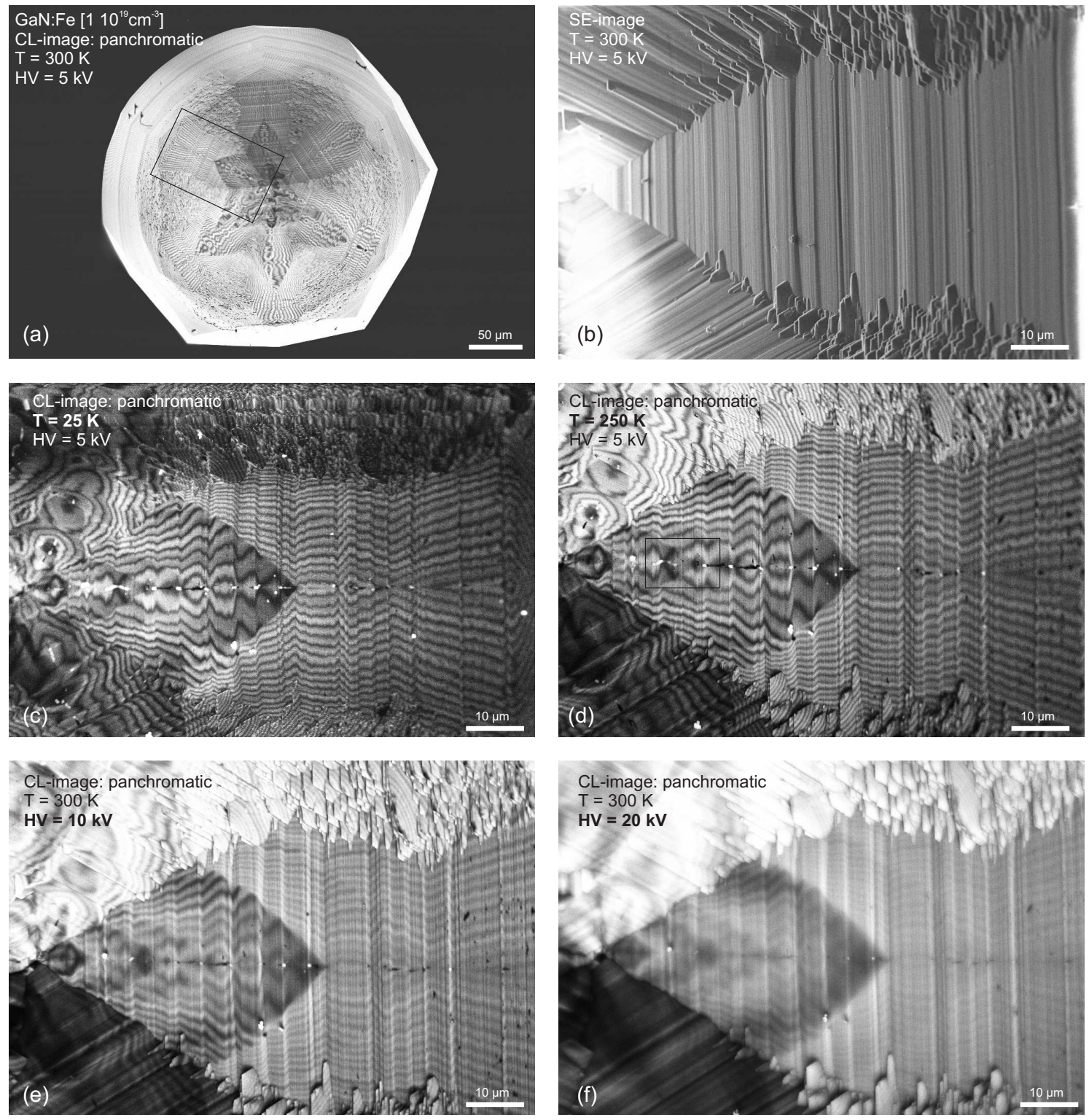

FIG. 3: Micrographs of the finely structured CL pattern that shows up at low acceleration voltage $\left(\mathrm{E}_{0}\right)$ in and around pits of Fe doped HVPE grown GaN $\left[1 \times 10^{19} \mathrm{~cm}^{-3}\right]$. (a) CL image of the same double pit as in Fig. 1 at HV $5 \mathrm{kV}$. The other images show the detail within the black frame. (b) SE image of the framed area. (c), (d) Temperature variation at $25 \mathrm{~K}$ and $250 \mathrm{~K}$, respectively. (e), (f) $\left(\mathrm{E}_{0}\right)$ variation at $10 \mathrm{kV}$ and $20 \mathrm{kV}$, respectively. 

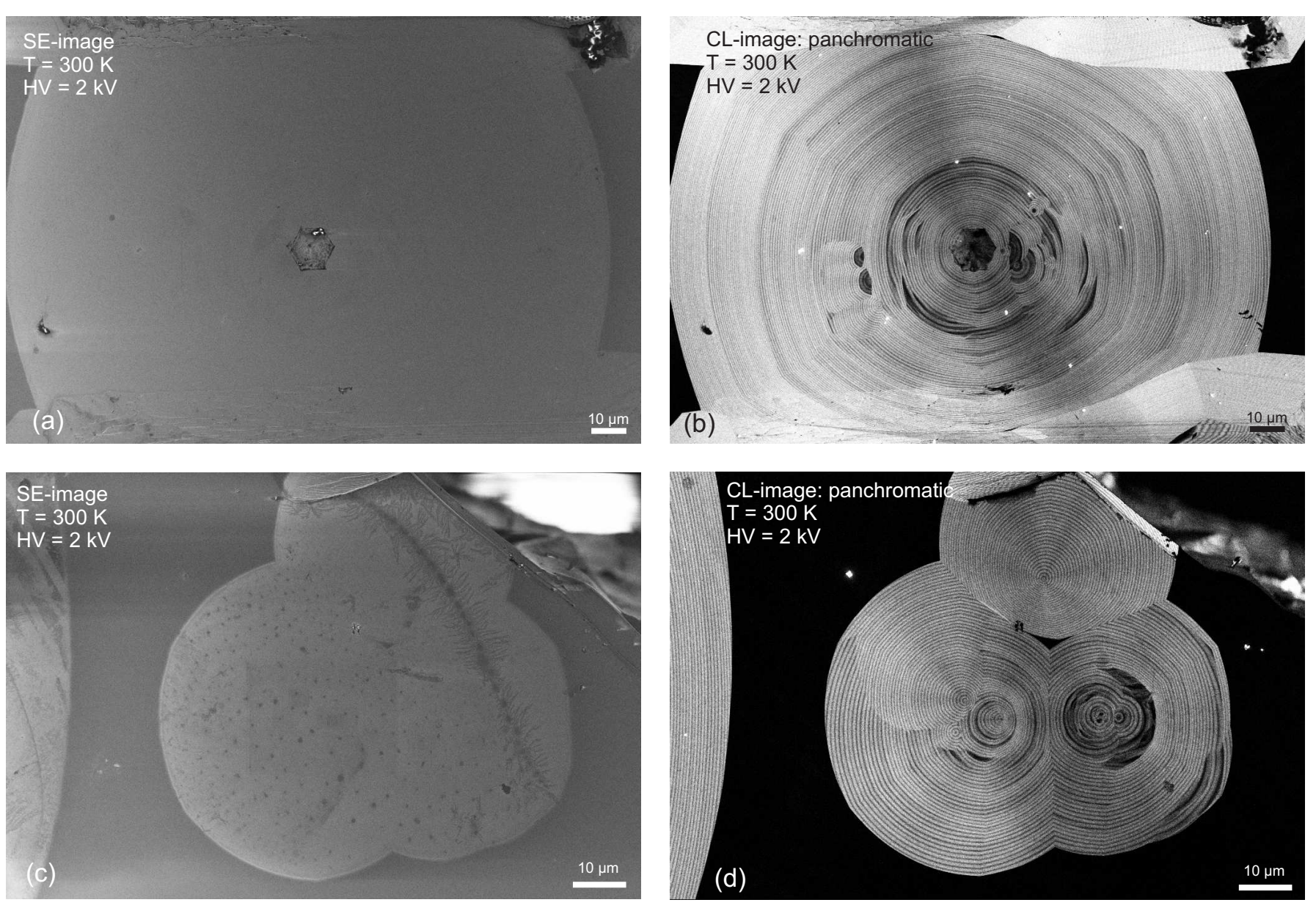

FIG. 4: SE and CL images of two different regions of the Fe doped HVPE GaN sample $\left[1 \times 10^{19} \mathrm{~cm}^{-3}\right]$. Both features consist of circular areas that appear slightly brighter in the $2 \mathrm{kV}$ SE image and that exhibit enhanced CL patterned in form of slightly faceted concentric circles. Only the circular area shown in (a) and (b) has a small pit in the center. 

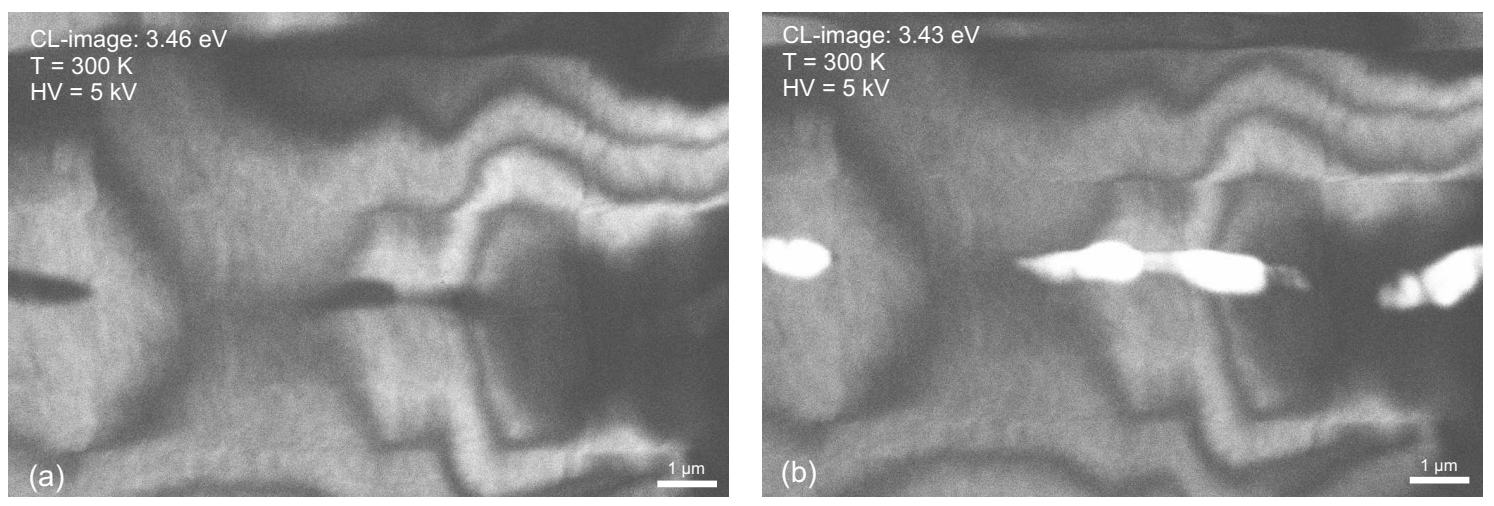

FIG. 5: Monochromatic CL images of the area round the symmetry axis running down the $11 \overline{2} 2$ facets of the pits (marked by a black frame in Fig. 3(d)). Image (a) and (b) were recorded for an emission energy of $3.46 \mathrm{eV}$ and $3.43 \mathrm{eV}$, respectively.

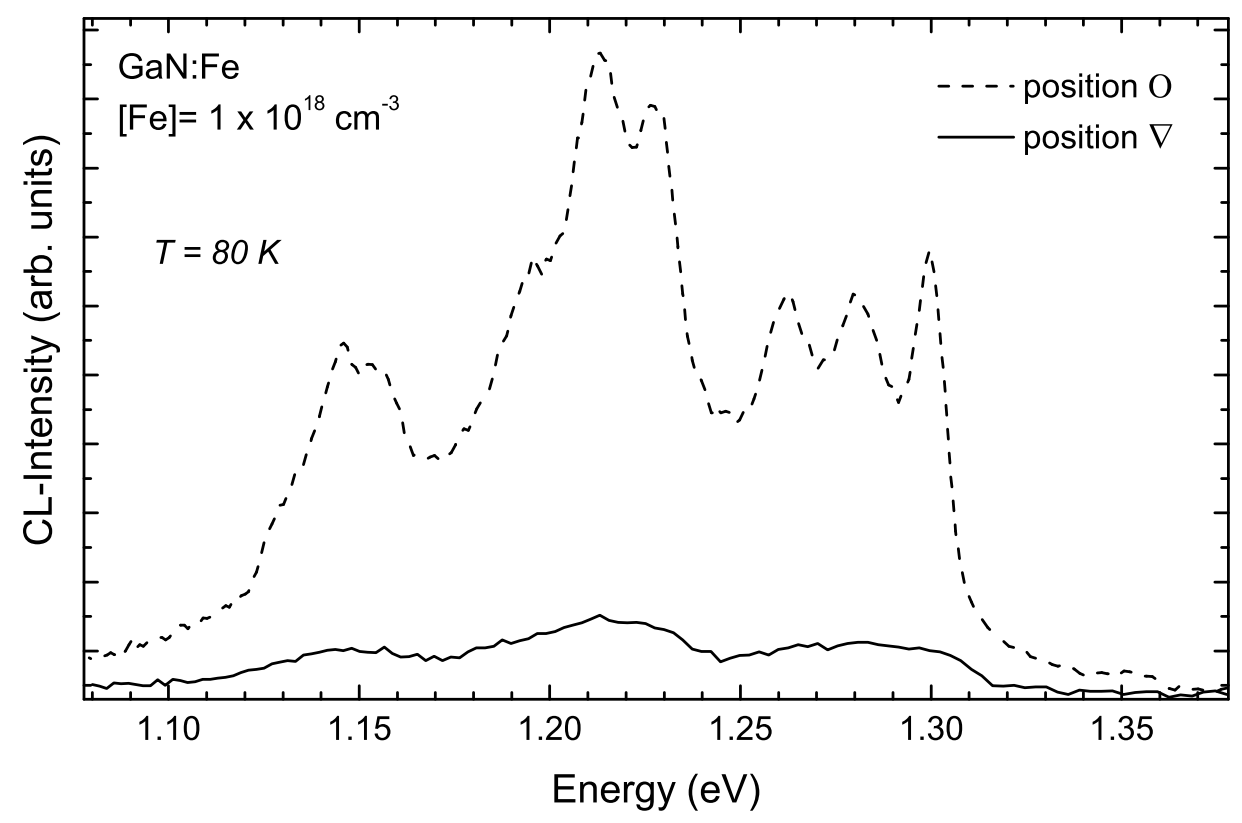

FIG. 6: Infrared CL spectra of Fe doped GaN $\left[1 \times 10^{18} \mathrm{~cm}^{-3}\right]$ at $80 \mathrm{~K}$. Acceleration voltage and current were $20 \mathrm{kV}$ and $5 \mathrm{nA}$, respectively. The complex emission structure originates from the intra-center ${ }^{4} \mathrm{~T}_{1}(\mathrm{G})-{ }^{6} \mathrm{~A}_{1}(\mathrm{~S})$ transition of $\mathrm{Fe}^{3+}$. The symbols $\nabla$ and $\bigcirc$ refer to Fig. 1 and indicate where the spectra were recorded, i.e., areas of strong or weak band-edge luminescence. 


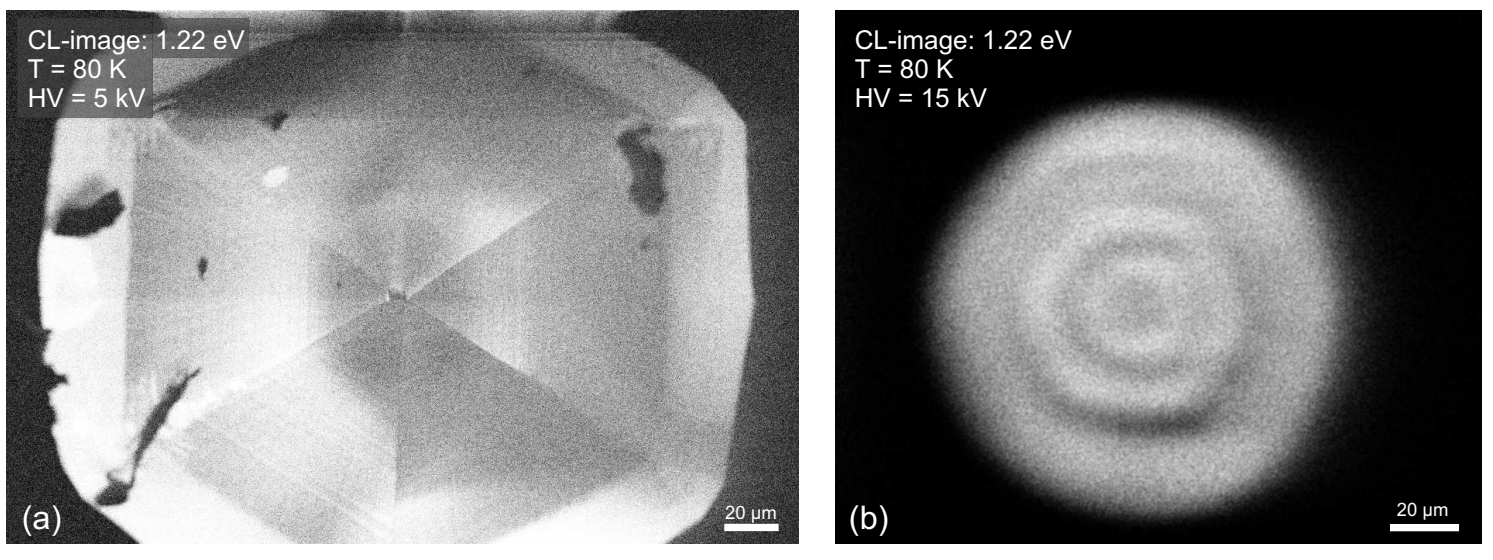

FIG. 7: Monochromatic CL images of Fe doped GaN $\left[1 \times 10^{19} \mathrm{~cm}^{-3}\right]$ at $1.22 \mathrm{eV}$ and $80 \mathrm{~K}$. Spatial distribution of $\mathrm{Fe}^{3+}$ luminescence. (a) Pit comparable to Fig. 1. (b) Area comparable to to Fig. 4(c),(d). 